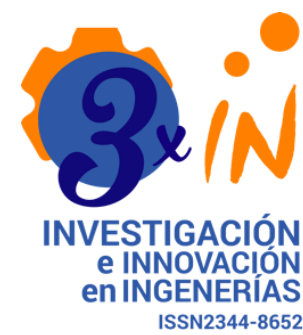

ISSN2344-8652

\title{
Identificación de perfiles característicos de las Tecnologías de la Información y Comunicación en empresas del sector manufacturero en Colombia mediante análisis de conglomerado y discriminante
}

\section{Identification of characteristic profiles of Information and Commu- nication Technologies in companies in the manufacturing sector in Colombia through cluster and discriminant analysis}

\author{
Efraín De La Hoz Granadillo iD, José Morelos Gómez \\ Universidad de Cartagena, Colombia \\ Diego Cardona Arbeláez \\ Universidad Libre Seccional Cartagena, Colombia
}

\begin{abstract}
Open Access
Recibido:

18 octubre de 2019

Aceptado:

10 diciembre de 2019

Publicado:

27 febrero de 2020

Correspondencia:

edelahozg@unicartagena.edu.co jmorelosg@unicartagena.edu.co diego.cardona@unilibrectg.edu.co
\end{abstract}

DOI:

https://doi.org/10.17081/invinno.8.1 .3676

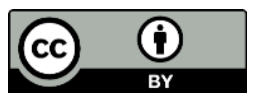

(c) Copyright: Investigación e Innovación en Ingenierías

\section{Resumen}

Objetivo: Estructurar un método para analizar la adopción y uso de las Tecnologías de la Información y la Comunicación-TIC en 7542 empresas del sector manufacturero de Colombia. Metodología: Para esta investigación se aplicaron las técnicas análisis multivariado de conglomerado y discriminante, y se tomaron los resultados de la encuesta anual manufacturera del Departamento Administrativo Nacional de Estadística de Colombia correspondiente al año 2017. Se aplicó un análisis de conglomerados para la identificación de perfiles característicos en la adopción y uso de las TIC, seguidamente se evaluó la capacidad de las variables analizadas para clasificar correctamente las empresas manufactureras en los perfiles identificados. Resultados: Los resultados muestran que el modelo clasifica correctamente en un $94,4 \%$ los perfiles TIC del sector manufacturero analizado. Conclusiones: La aplicación del método permitió contrastar la capacidad discriminante de las variables para identificar perfiles característicos significativamente homogéneos intra-grupos y heterogéneos extra-grupos.

Palabras claves: Tecnologías de la información y comunicación, análisis de conglomerado, análisis discriminante y perfiles característicos.

\section{Abstract}

Objective: Structure a method to analyze the adoption and use of Information and Communication Technologies-ICT in 7542 companies in the Colombian manufacturing sector. Methodology: For this research, the multivariate analysis of conglomerate and discriminant techniques were applied, and the results of the annual manufacturing survey of the National Administrative Department of Statistics of Colombia for 2017 were applied. A cluster analysis was applied to identify characteristic profiles in the adoption and use of ICT, then the ability of the analyzed variables to correctly classify the manufacturing companies in the profiles identified was evaluated. Results: The results show that the model correctly classifies the ICT profiles of the analyzed sector in $94.4 \%$. Conclusions: The application of the method allowed to contrast the discriminant capacity of the variables to identify characteristic profiles that were significantly homogeneous intra-groups and heterogeneous extra-groups.

Keywords: Information and communication technologies, conglomerate analysis, discriminant analysis and characteristic profiles.
Como citar (IEEE): E. De La Hoz-Granadillo, J. Morelos Gómez., y D. Cardona Arbeláez, “Identificación de perfiles característicos de las Tecnologías de la Información y Comunicación en empresas del sector manufacturero en Colombia mediante análisis de conglomerado y discriminante", Investigación e Innovación en Ingenierías, vol. 8, n. 1, 2020. DOI: https://doi.org/10.17081/invinno.8.1.3676 


\section{Introducción}

El análisis de los perfiles competitivos de un clúster empresarial o sector económico en particular, se ha convertido en un propósito estratégico para la proyección de las empresas, mejorar la ventaja competitiva y la toma de decisiones gerenciales que contribuyan al mejoramiento de eficacia y la eficiencia de las actividades operacionales y administrativas [1].

Es este sentido, la identificación de los perfiles característicos de las tecnologías de la información y comunicación (TIC) para el sector manufacturero en Colombia, reviste gran importancia dado los requerimientos tecnológicos que facilitan el procesamiento de la información y comunicación interna, los cuales se hacen necesarios su aplicación para facilitar el análisis de los datos de las actividades de las áreas relacionadas con el fin de diseñar las estrategias competitivas que contribuyan al crecimiento competitivo de dicho sector.

Estudios similares muestran la pertinencia en el ámbito internacional de la aplicación de las TIC en empresas que orientan sus propósitos hacia el mejoramiento de la competitividad en sectores manufactureros en Barbados y España [2,3], los cuales identificaron que existe un consenso sobre las ventajas y oportunidades de uso de las variables que promueven las TIC, particularmente, en la rapidez con que se responde a los cambios internos de la organización y del mercado competitivo.

En este sentido, otras investigaciones han trabajado en la concepción de perfiles en el sector empresarial, como lo señalan diferentes autores [4, 5]. Asimismo, algunos autores aplicaron la estadística multivariante en el sector de las pymes para evaluar su comportamiento.

En razón de todo lo expuesto, esta investigación busca identificar los perfiles característicos de las empresas de las tecnologías de la información y comunicación pertenecientes al sector manufacturero, para lo cual se busca dar respuesta a los siguientes interrogantes problémicos. ¿Cuáles son los variables que permiten determinar perfiles TIC en las empresas manufactureras de Colombia?, ¿Que técnicas de análisis multivariado permiten identificar perfiles TIC empresariales?, ¿Cómo determinar los perfiles TIC del sector manufacturero?, ¿Cómo valorar la consistencia de asociación de los perfiles TIC?, ¿Cómo establecer rangos en los perfiles TIC de las organizaciones objeto de estudio?, ¿Qué capacidad discriminante tiene las variables analizadas para los perfiles TIC de las empresas manufactureras?.

Para dar respuesta a los interrogantes de investigación, se desarrolló un análisis racional-empírico, que permitió definir las variables y criterios de clusterización de 7542 empresas del sector manufacturero que respondieron la encuesta anual Manufacturera 2017 del Departamento Administrativo Nacional de Estadística -DANE. Con las variables y datos 
seleccionados, se aplicó la técnica de análisis de conglomerado para identificar perfiles característicos TIC. Asimismo, el número de clúster discriminantes del sector se determinó mediante el análisis del nivel de distancia entre grupos.

Para el desarrollo de esta investigación, se realizó una descripción de los principales referentes teóricos que aportan al tema de investigación. la metodología utilizada, describe el tipo de investigación, técnica estadística, población, fuentes y datos para identificar los perfiles característicos de las tecnologías de información y comunicación en el sector manufacturero en Colombia; finalmente, se presentan los resultados obtenidos referentes al análisis de conglomerados y clasificación del modelo del modelo discriminante en el sector objeto de estudio.

\section{Marco referencial}

El mundo empresarial moderno no es ajeno hoy a los volátiles cambios a los que se ven enfrentado las empresas, variaciones éstas que ser relacionan con variables de tipo económico, de mercadeo, logístico, estratégico y tecnológico. Siendo este último, particularmente, el relacionado con las tecnologías información y la comunicación (TIC) [6].

Sin duda, esta incertidumbre de mercado se ha convertido en un desafío clave para clave para los líderes empresariales, que tienen la responsabilidad preparar las organizaciones para enfrentar las variaciones y cambios no controlables del mercado competitivo. Esto es particularmente desafiante cuando se trata de las TIC que pueden interrumpir la administración de los sistemas de información empresarial o los procesos de negocios existentes restando valor a las actividades operacionales y de gestión organizacional [7].

En este sentido la aplicación de las TIC, como modelo tecnológico de negocio viene tomando importancia como una herramienta efectiva que las organizaciones pueden usar para prepararse para el cambio relacionado con las tecnologías de la información y la comunicación [8]. Sin embargo, existe evidencia que demuestra que el uso de las TIC no todas las veces resulta ventajoso dado los comportamientos deliberados e intencionado de algunos colaboradores que interfieren y limitan el mejoramiento de los procesos productivos y gerenciales del clúster empresarial $[9,10]$

Impulsar el mejoramiento de las organizaciones, requiere esencialmente de la aplicación de herramientas de gestión que faciliten el desarrollo de las acciones y planes para incrementar la ventaja la competitividad de una empresa. Para lograr lo anterior, se requiere comparar el perfil competitivo de la compañía con un estándar competitivo modelo óptimo de competitividad, con el propósito de poder establecer las brechas y las 
estrategias coadyuvaran al mejoramiento del perfil e incremento del ranking competitivo $[11,12]$.

\section{Análisis de conglomerado y discriminante}

El análisis de conglomerados o de clúster es una técnica estadística multivariada que permite identificar la homogeneidad de las variables seleccionadas considerando las similitudes o características comunes en el grupo de observaciones analizadas [13]. Es utilizado en el diseño de estructuras jerárquicas, agrupación de observaciones o variables [13]. Para lo anterior, el análisis de clúster desarrolla un análisis de similitud de las observaciones para facilitar el agrupamiento.

En la clasificación de variables comúnmente es utilizado el coeficiente de Pearson cuadrado para medidas de similaridad. Y para determinar la disimilaridad es utilizada la distancia euclídea al cuadrado, como se muestra en la ecuación 1 . Siendo $d^{2}{ }_{i j}$ la distancia entre las muestras $i$ y $j, r$ el total de variables, $X_{i k}$ el valor de la observación $i$ para cada variable $k, X_{j k}$ el valor de la observación $j$ para la variable $k$.[14].

$$
d^{2}{ }_{i j}=\sum_{k=1}^{r}\left(X_{i k}-X_{j k}\right)^{2}
$$

En el encadenamiento se integran observaciones para obtener grupos homogéneos [14]. De los criterios y medidas analizadas en la literatura, el método de Ward se caracteriza por reducir la varianza en la integración de grupos homogéneos [15]. Las ecuaciones 2 y 3 , muestran el cálculo de estos parametros

$$
\begin{aligned}
& W=\sum_{g=1}^{G} \sum_{i=1}^{n_{g}}\left(X_{i g}-\overline{X_{g}}\right)\left(X_{i g}-\overline{X_{g}}\right)^{\prime} \\
& \min \frac{n_{a} n_{b}}{n_{a}+n_{b}}\left(\overline{X_{a}}-\overline{X_{b}}\right)^{\prime}\left(\overline{X_{a}}-\overline{X_{b}}\right)
\end{aligned}
$$

Siendo $X_{i g}$ la variable que proporciona el valor del individuo $i$ en el grupo $g$, $\overline{X_{g}}$ el promedio de la variable en el grupo $g, n_{g}$ el total de individuos del grupo $g, G$ el total de grupos. La ecuación 3 es utilizada para seleccionar los grupos a fusionar, siendo $n_{a} y n_{b}$ el total de observaciones en cada grupo de cada grupo a fusionarse, y $\overline{X_{a}} y \overline{X_{b}}$ el valor promedio de cada grupo [13]: 
El Análisis Discriminante Multivariado corresponde a las llamadas técnicas de clasificación supervisadas[16]. La técnica utiliza información de los elementos asociados a clases para calcular funciones discriminantes, con capacidad de clasificar una observación en una clase específica. También es aplicada en la reducción del número de variables en problemas con gran número de variables, datos u observaciones:

Sea $\vec{X}=(\mathrm{X} 1, \mathrm{X} 2, \ldots, \mathrm{Xp})$ un vector con $\mathrm{p}$ variables aleatorias del problema, entonces se define $Y_{i}$ la función discriminante a partir de la combinación lineal de variables originales $X 1, X 2, \ldots X p$, de manera que se minimice la variación intra grupal y se maximice la variación entre grupo. Ver ecuación 5 , con $\beta_{i 0}$ una constante, $\beta_{i j}$ ponderación discriminante de la variable independiente $X_{j}, \mathrm{j}=1, \ldots, \mathrm{p}$.

$$
\begin{aligned}
& Y_{i}=\beta_{i 0}+\beta_{i 1} X_{1}+\cdots+\beta_{i p} X p \\
& \mathrm{i}=1, \ldots, \min \{\mathrm{p}, \mathrm{g}-1\}
\end{aligned}
$$

De igual manera, investigaciones desarrolladas en clústeres de negocios similares igualmente han utilizado la técnica discriminante para validar las diferencias significativas entre grupos de analizados [17].

\section{Metodología}

Para este artículo de investigación, se desarrolló un análisis racionalempírico, que permitió definir las variables y criterios de clusterización y discriminación del modelo en 7542 empresas del sector manufacturero de Colombia que respondieron la encuesta anual Manufacturera 2017 del Departamento Administrativo Nacional de Estadística -DANE.

Las características seleccionadas se relacionan con la adquisición y uso de las TIC del sector manufacturero (computador de escritorio, computador portátil, tableta, teléfono inteligente, PDA-Asistente personal digital, aplicaciones y/o programas informáticos). Con las variables y datos se utilizó el análisis conglomerado para identificar Perfiles característicos TIC en empresas del sector manufacturero en Colombia para lo cual se aplicaron y evaluaron medidas de distancia y métodos de vinculación criterios de agrupación disponibles en el software Minitab 18.

El número de clúster discriminantes del sector se determinó mediante el análisis del nivel de distancia entre grupos. Asimismo, se evaluó el nivel de discriminación de los perfiles TIC, apoyados con en el software IBM SPSS Stastistic 25, con lo cual se analizó el nivel de discriminación de los perfiles tecnológicos del sector manufacturero. Con resultados estadísticos, se estableció la capacidad discriminante de las variables analizadas, así como la 
estadística de grupos con lo cual se caracterizaron los perfiles TIC del sector empresarial manufacturero.

En la figura 1, se presenta el esquema del método para definir perfiles TIC de las empresas del sector manufacturero.

Figura 1. Método para definir perfiles TIC en empresas manufactureras

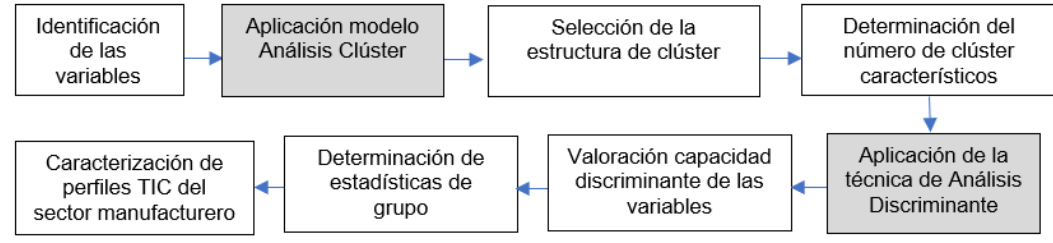

Fuente: Elaboración propia

\section{Resultados y discusión}

Con los datos de la Encuesta Anual Manufacturera de las Tecnologías de la Información y Comunicaciones-EAM-TIC 2017, asociados a la adquisición y uso de las TIC en las empresas se aplicó un análisis de conglomerado, en el que se seleccionó el modelo con criterio de agrupamiento de Ward y medida de similitud la distancia euclidiana con cuatro conglomerados, por presentar mejores niveles de homogeneidad intragrupo y heterogeneidad extragrupo, como se observa en la figura 2 , del dendograma de conglomerados de perfiles TIC en empresas del sector manufacturero y el nivel de distancias TIC, el cual muestra la conveniencia de seleccionar 4 grupos o perfiles TIC.

Figura 2. Dendograma de conglomerados TIC en empresas manufactureras

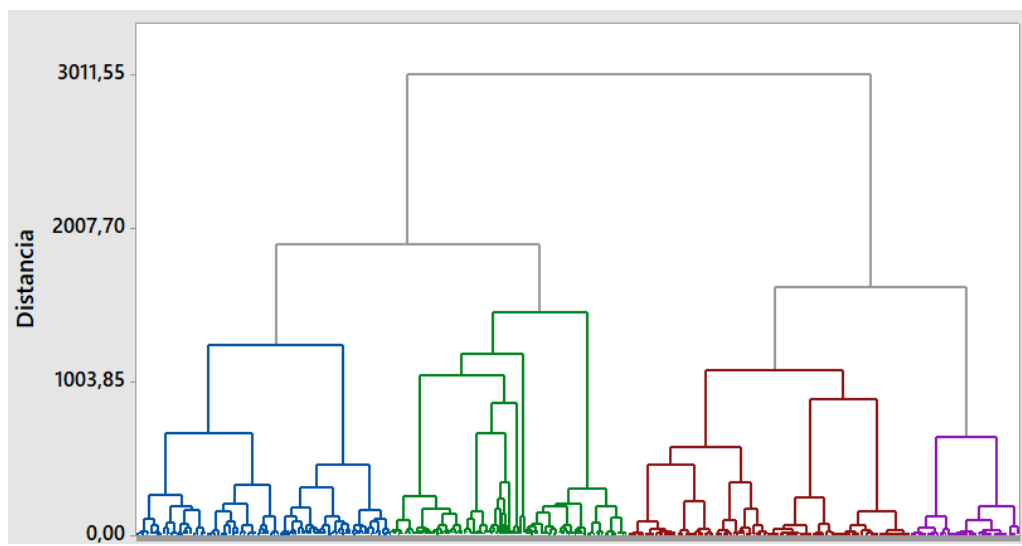

Fuente: Elaboración propia

En relación con los datos de la Tabla 1, se observan los resultados de distancia máxima y promedio en los centroides de los grupos TIC, con una distancia promedio 
de 2,7351425, desde el centroide, asociado a un alto nivel de homogeneidad entre los grupos.

Tabla 1. Distancia máxima y promedio de los centroides para los perfiles TIC

\begin{tabular}{|l|c|c|c|c|}
\hline & $\begin{array}{c}\text { Número de } \\
\text { observaciones }\end{array}$ & $\begin{array}{c}\text { Dentro de la } \\
\text { suma de } \\
\text { cuadrados del } \\
\text { conglomerado }\end{array}$ & $\begin{array}{c}\text { Distancia } \\
\text { promedio } \\
\text { desde el } \\
\text { centroide }\end{array}$ & $\begin{array}{c}\text { Distancia } \\
\text { máxima desde } \\
\text { centroide }\end{array}$ \\
\hline Conglomerado1 & 2182 & 17887,9 & 2,83841 & 4,9989 \\
\hline Conglomerado2 & 2411 & 8227,7 & 1,78469 & 4,2609 \\
\hline Conglomerado3 & 2013 & 92501,6 & 5,11963 & 66,4085 \\
\hline Conglomerado4 & 936 & 1533,9 & 1,19784 & 2,9343 \\
\hline \multicolumn{3}{|c|}{ Promedio } & 2,7351425 & 19,65065 \\
\hline
\end{tabular}

Fuente: Elaboración propia

En la Tabla 2, se presentan los trayectos promedio de los centroides para cada perfil o conglomerado. Asimismo, se observa el nivel de heterogeneidad con 2,954358 de distancia promedio entre centroides.

Tabla 2. Distancia entre perfiles TIC en empresas manufactureras

\begin{tabular}{|l|c|c|c|c|}
\hline & Conglomerado1 & Conglomerado2 & Conglomerado3 & Conglomerado4 \\
\hline Conglomerado1 & 0,00000 & 2,25540 & 2,97399 & 2,93855 \\
\hline Conglomerado2 & 2,25540 & 0,00000 & 3,11003 & 2,32272 \\
\hline Conglomerado3 & 2,97399 & 3,11003 & 0,00000 & 4,12546 \\
\hline Conglomerado4 & 2,93855 & 2,32272 & 4,12546 & 0,00000 \\
\hline
\end{tabular}

Fuente: Elaboración propia

De los resultados anteriores, cabe anotar que en el análisis de conglomerados se utilizaron los criterios de medidas de distancia incluidos en el software minitab 18 . Los mejores resultados de clasificación se obtuvieron con el criterio de Ward y la medida de distancia euclidiana.

En la Tabla 3, se muestran las medias y varianza de las variables TIC que caracterizan los 4 perfiles identificados en el análisis.

Tabla 3.Caracterización de perfiles TIC empresas manufactureras

\begin{tabular}{|c|c|c|c||c|c|c|c|}
\hline Variable* $^{*}$ & Grupo & Media & Varianza & Variable & Grupo & Media & Varianza \\
\hline \multirow{3}{*}{ B_1_A } & 1 & 1,00 & 0,000000 & B_1_6 & 1 & 21,60 & 1303,083 \\
\cline { 2 - 5 } & 2 & 1,00 & 0,000000 & & 2 & 18,03 & 487,500 \\
\cline { 2 - 5 } & 3 & 1,06 & 0,0565 & & 3 & 136,11 & 101563,86 \\
\cline { 2 - 7 } & 4 & 1,00 & 0,000000 & & 4 & 5,71 & 45,840 \\
\hline B_1_B & 1 & 1,30 & 0,2105 & B_2 & 1 & 39,34 & 661,261 \\
\hline
\end{tabular}


Identificación de perfiles característicos de las Tecnologías de la Información y Comunicación en empresas del sector manufacturero en Colombia mediante análisis de conglomerado y discriminante

\begin{tabular}{|c|c|c|c|c|c|c|c|}
\hline & 2 & 1,10 & 0,0900 & & 2 & 41,00 & 683,552 \\
\hline & 3 & 1,08 & 0,0819 & & 3 & 51,90 & 886,584 \\
\hline & 4 & 2,00 & 0,000000 & & 4 & 28,32 & 490,514 \\
\hline \multirow[t]{4}{*}{ B_1_C } & 1 & 1,99 & 0,000458 & \multirow[t]{4}{*}{ B_2_1 } & 1 & 1,51 & 0,2499 \\
\hline & 2 & 2,00 & 0,000000 & & 2 & 1,00 & 0,000000 \\
\hline & 3 & 1,33 & 0,2238 & & 3 & 1,19 & 0,1548 \\
\hline & 4 & 2,00 & 0,000000 & & 4 & 1,00 & 0,000000 \\
\hline \multirow[t]{4}{*}{ B_1_D } & 1 & 1,37 & 0,2341 & \multirow[t]{4}{*}{ B_2_2 } & 1 & 1,32 & 0,2204 \\
\hline & 2 & 1,38 & 0,2359 & & 2 & 1,99 & 0,000829 \\
\hline & 3 & 1,25 & 0,1905 & & 3 & 1,50 & 0,2501 \\
\hline & 4 & 1,63 & 0,2310 & & 4 & 2,00 & 0,000000 \\
\hline \multirow[t]{4}{*}{ B_1_E } & 1 & 2,00 & 0,000000 & \multirow[t]{4}{*}{ B_2_3 } & 1 & 2,00 & 0,000000 \\
\hline & 2 & 2,00 & 0,000000 & & 2 & 2,00 & 0,000000 \\
\hline & 3 & 1,87 & 0,1103 & & 3 & 1,58 & 0,2421 \\
\hline & 4 & 2,00 & 0,000000 & & 4 & 2,00 & 0,000000 \\
\hline \multirow[t]{4}{*}{ B_1_1 } & 1 & 12,64 & 440,088 & \multirow[t]{4}{*}{ B_2_4 } & 1 & 1,56 & 0,2463 \\
\hline & 2 & 10,93 & 197,563 & & 2 & 2,00 & 0,000000 \\
\hline & 3 & 58,81 & 14806,34 & & 3 & 1,70 & 0,2089 \\
\hline & 4 & 4,872 & 37,650 & & 4 & 2,00 & 0,000000 \\
\hline \multirow[t]{4}{*}{ B_1_2 } & 1 & 4,522 & 127,930 & \multirow[t]{4}{*}{ B_3 } & 1 & 1,00 & 0,000000 \\
\hline & 2 & 3,770 & 63,113 & & 2 & 1,00 & 0,000000 \\
\hline & 3 & 39,66 & 13800,42 & & 3 & 1,01 & 0,0195 \\
\hline & 4 & 0,000 & 0,000000 & & 4 & 1,00 & 0,000000 \\
\hline \multirow[t]{4}{*}{ B_1_3 } & 1 & 0,0027 & 0,01650 & \multirow[t]{4}{*}{ B_3_1 } & 1 & 1,00 & 0,000000 \\
\hline & 2 & 0,00 & 0,000000 & & 2 & 1,00 & 0,000000 \\
\hline & 3 & 6,37 & 815,048 & & 3 & 0,98 & 0,02412 \\
\hline & 4 & 0,00 & 0,000000 & & 4 & 1,00 & 0,000000 \\
\hline \multirow[t]{4}{*}{ B_1_4 } & 1 & 4,435 & 128,537 & \multirow[t]{4}{*}{ B_3_2 } & 1 & 1,55 & 0,2472 \\
\hline & 2 & 3,340 & 39,327 & & 2 & 1,56 & 0,2463 \\
\hline & 3 & 25,52 & 7255,06 & & 3 & 1,3 & 0,2513 \\
\hline & 4 & 0,84 & 3,3895 & & 4 & 2,0 & 0,000000 \\
\hline \multirow[t]{4}{*}{ B_1_5 } & 1 & 0,00 & 0,000000 & & & & \\
\hline & 2 & 0,00 & 0,000000 & & & & \\
\hline & 3 & 5,75 & 3348,01 & & & & \\
\hline & 4 & 0,00 & 0,000000 & & & & \\
\hline
\end{tabular}

*La variables se identifican y operacionalizan en el informe Encuesta Anual Manufacturera-EAM-TIC 2017

Fuente: [18]

Con los resultados obtenidos en la técnica de clúster se validar el poder de clasificación de los factores analizados, lo cual permite determinar los perfiles TIC, lo que posibilita el análisis de perfiles en la disposición y uso TIC en la operación de las empresas manufactureras colombianas, con el fin de 
caracterizar el sector y comprender las condiciones de desarrollo tecnológico (Ver Tabla 4).

Por otra parte, los resultados del análisis discriminante multivariado, muestra un $94.4 \%$ de clasificación correcta, lo cual se asume como una capacidad de discriminación excelente. Lo anterior, justifica el nivel de homogeneidad intragrupo y heterogeneidad extragrupo de los perfiles TIC del sector manufacturero.

Tabla 4.Clasificación correcta de perfiles TIC

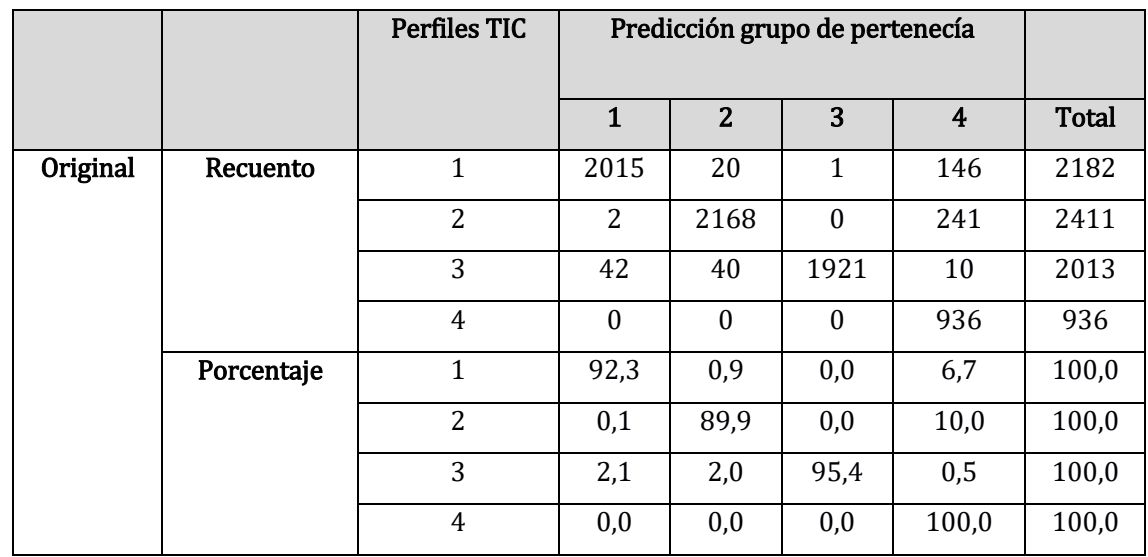

Fuente: Elaboración propia

Con el uso de técnica discriminante esta investigación se comprueba la capacidad de discriminación de las variables analizadas en el sector manufacturero de Colombia. A partir de las variables relacionadas uso de: computador de escritorio, computador portátil, tableta, teléfono inteligente, PDA-Asistente personal digital, aplicaciones y/o programas informáticos asociados a las tecnologías de información y comunicación de las empresas. Con lo cual se muestra la pertinencia del método propuesto en la Figura 1.

De otro lado, es importante señalar que otros estudios con metodologías similares para analizar perfiles empresariales, contrastan la robustez de la técnica de conglomerado en sectores empresariales similares [19, 20, 21, 22, 23].

En este mismo sentido, otros estudios abordan la conformación de clústeres empresariales a partir de otras variables significativas como la cooperación, el conocimiento e innovación y las redes sociales, para analizar su influencia en la competitividad como se hace en esta investigación [24, 25].

\section{Conclusiones}

De manera general en esta investigación se estructuró un método para analizar la adopción y uso de las Tecnologías de la Información y la Comunicación-TIC mediante la articulación de técnicas de análisis 
multivariado, con lo cual se aporta en los procesos de análisis de variables y resultados de la gestión tecnológica aplicados en el contexto del sector de telecomunicaciones. A partir de las variables asociadas al número de computadores de escritorio, computadores portátiles, tabletas, teléfonos inteligentes, PDA-Asistente personal digital, aplicaciones y/o programas informáticos asociados a las tecnologías de información y comunicación de las empresas, las cuales fueron tomadas de la encuesta anual manufacturera del Departamento Administrativo Nacional de Estadística de Colombia, se evidencia la pertinencia de las variables y la pertinencia de la técnica multivariada de análisis de conglomerado o clúster para establecer grupos identificados en esta investigación como perfiles característicos de las Tecnologías de la Información y Comunicación con lo cual se valora la aplicación de esta técnica para mejorar la comprensión, análisis y toma de decisiones gerenciales en función del análisis de las variables objeto de investigación. Así mismo, la integración del análisis de conglomerados con la técnica de análisis discriminante, permitió establecer una herramienta para valorar la capacidad discriminante de las variables para identificar grupos significativamente homogéneos intra-grupos y heterogéneos extragrupos. Como líneas futuras de investigación, se propone extender la aplicación de este método de análisis en otros sectores empresariales a partir de variables asociadas a los procesos de gestión y sus resultados.

\section{Referencias bibliográficas}

1. J. Morelos, T.J. Fontalvo, and E. De la Hoz, "Behaviour of productivity indicators and financial resources in the field of extraction and exploitation of minerals in Colombia", International Journal of Productivity and Quality Management, Vol. 25, $\mathrm{n}^{\circ}$. 3, pp. 349-367, 2018. DOI: https://doi.org/10.1504/IJPQM.2018.095651.

2. L. Seon, S. Mandhi, and W. Kelly, "Information and communication technology strategies to improve international competitiveness in the wholesale and retail trade sector", International Journal Business and globalization, Vol. 20, no. 1, pp. 128-138, 2018. DOI: https://doi.org/10.1504/IJBG.2018.088680

3. M.E. Ruiz, I. Gil, and B. Moliner, "Information and communication technologies in rural hotels", International Journal of Sustainable Economy, Vol. 2, $\mathrm{n}^{\circ}$. 1, pp. 1-15. 2009. DOI: https://doi.org/10.1504/IJSE.2010.029938

4. E. De La Hoz, A. González, and A. Santana, "Metodología de Medición del Potencial Exportador de las Organizaciones Empresariales", Información tecnológica, Vol. 27, nº. 6, pp. 11- 
18. 2016. DOI: http://dx.doi.org/10.4067/S071807642016000600003

5. E. De La Hoz, T.J. Fontalvo, and J. Morelos, "Diseño de Perfiles Financieros Empresariales del Sector Químico en Colombia mediante Cálculo Multivariado", Información tecnológica, Vol. 29, $\mathrm{n}^{\circ}$. 4, pp. 197-204, 2018. DOI: http://dx.doi.org/10.4067/S0718-07642018000500227

6. T. Clohessy, T. Acton, and L. Morgan, "(De) Mystifying the information and communication technology business model concept", International Journal of Networking and Virtual Organisations, Vol. 20, $\mathrm{n}^{\circ}$. 4, pp. 377-399, 2019. DOI: https://doi.org/10.1504/IJNV0.2019.100599

7. K. Phornlaphatrachakorn, "Influences of strategic management accounting on firm profitability of information and communication technology businesses in Thailand", International Journal of Business Excellence, Vol. 17, $\mathrm{n}^{\circ} .2$, $\mathrm{pp}$. 131-153. 2019. DOI: https://doi.org/10.1504/IJBEX.2019.097540

8. P. Neirotti, M. Cantamessa, and E. Paolucci, "Do companies with a competitive advantage make better use of IT? Evidence from Italian enterprises", International Journal of Technology Management, Vol. 42. $\mathrm{n}^{\circ}, 1-2$, pp. 158-184, 2008. DOI: https://doi.org/10.1504/IJTM.2008.018066

9. T. Lin, and K. Lin. "The negative effects of using information and communication technology in a Taiwanese enterprise", International Journal of Enterprise Network Management, Vol. 2, $\mathrm{n}^{\circ}$. 3, pp. 318-332, 2008. DOI:https://doi.org/10.1504/IJENM.2008.018784

10. C. Antonelli, A. Geuna, and W. E. Steinmueller, "Information and communication technologies and the production, distribution and use of knowledge", International Journal of Technology Management, Vol. 20, $\mathrm{n}^{\circ}$. 1-2, pp. 72-94, 2000. DOI:https://doi.org/10.1504/IJTM.2000.002853

11. W. C. Lucato, M. Vieira, and J.D. Filho, "The competitive distance as a tool for competitiveness improvement", International Journal of Business Excellence, Vol. 8, n.2, pp. 181-196, 2015. DOI: https://doi.org/10.1504/IJBEX.2015.068207

12. M. Cesário, and T. De Noronha, "Regional, national and international networks: the suitability of different competitive strategies for different geographic profiles", 15th Uddevalla Symposium 2012. Entrepreneurship and Innovation networks. University West, 2012. DOI:https://doi.org/10.1504/IJESB.2014.060895

13. D. Peña, Análisis de datos multivariantes. Madrid: Editorial Mc.Graw Hill, 2002, pp. 137-235. 
14. J. De la Garza, B. Morales, and B. González, Análisis Estadístico Mutivariante. Un enfoque teórico y práctico. México: Editorial Mc.Graw Hill, 2013, pp. 150-178.

15. A. Catena, M. Ramos, and H. Trujillo, Análisis Multivariado. Un manual para investigadores. Madrid: Editorial Biblioteca Nueva, 2003.

16. Z. Lai, Y. Xu, J. Yang, J. Tang, and D. Zhang, "Sparse tensor discriminant analysis," IEEE Trans. Image Process., vol. 22, n. 10, pp. 3904-3915, 2013.

17. J. Morelos, E. De La Hoz, and T. J. Fontalvo, "Método de cálculo multivariante para analizar y proyectar el comportamiento de las razones financieras de grupos empresariales del sector extracción en Colombia." Interciencia, Vol. 43. n. 10.pp. 696-700. 2018.

18. Dane, "COLOMBIA - Encuesta Anual Manufacturera - EAM - 2016 - 2017," 2017.

19. J. Aznar, R.E. Cervelló, and A. Romero, "New spanish banking conglomerates. Application of the analytic hierarchy process (AHP) to their market value." International Research Journal of Finance and Economics, Vol. 78, $\mathrm{n}^{\circ}$. 7, pp. 1-82. 2011. DOI:http://hdl.handle.net/10251/35967

20. J. Aznar, R. E. Cervelló, and F. García, "Spanish savings banks and their future transformation into private capital banks. determining their value by a multicriteria valuation methodology." European Journal of Economics, Finance and Administrative Sciences, Vol. 35, no. 1, pp. 156-164. 2011. DOI:http://hdl.handle.net/10251/35857

21. E. De la Hoz, T. Fontalvo, and J. Morelos, "Evaluación del Comportamiento de los Indicadores del Sector Petróleo y Gas en Colombia Mediante el Análisis Discriminante, Contaduría y administración, Vol. 59, $\mathrm{n}^{\circ}$. 4, pp. 167-191, 2014. DOI: https://doi.org/10.1016/S0186-1042(14)70159-7

22. F. García, F. Guijarro, and I. Moya, "Ranking Spanish savings banks: A multicriteria approach", Mathematical and computer modelling, Vol. 52, no. 7-8, pp. 1058-1065, 2010. DOI: https://doi.org/10.1016/j.mcm.2010.02.015

23. Y. J. Wang, and $H$. Lee, "A clustering method to identify representative financial ratios." Information Sciences, Vol. 178, no. 4, pp. 1087-1097. 2008. DOI: https://doi.org/10.1016/j.ins.2007.09.016

24. F. Becerra, H. M. Serna, and J. C. Naranjo, "Redes empresariales locales, investigación y desarrollo e innovación en la empresa. Cluster de herramientas de Caldas, Colombia", Estudios Gerenciales, Vol. 29, n. 127, pp. 247-257, 2013. 
25. J. Gómez, "La importancia de los factores fundamentales en el cluster." Dimensión Empresarial, Vol. 15, n.2, pp. 141-153, 2017. DOI: https://doi.org/10.15665/rde.v15i2.917 